\title{
Adjustment of relative gravity measurements using weighted and datum-free constraints is
}

\author{
Cheinway Hwang*, Cheng-Gi Wang, Li-Hua Lee \\ Department of Civil Engineering, National Chiao Tung University, 1001 Ta Hsueh Road, Hsinchu 300, Taiwan
}

Received 19 April 2001; received in revised form 27 November 2001; accepted 10 December 2001

\begin{abstract}
A program in FORTRAN 90 is developed which can adjust relative gravity measurements and solve for gravimeter parameters using the weighted constraint and datum-free constraint models. The weighted constraint model is chosen when there exist reliable a priori gravity values for use as supplementary data, or when it is required that a gravity network be attached to an existing gravity network of a higher order. The datum-free model uniquely determines relative gravity values among all stations without the need of a fixed gravity value, thus it is suitable for detecting relative gravity change. The optional solve-for gravimeter parameters include drift coefficients, and coefficients of the long wavelength and periodic components of calibration function. The program can also detect outliers in observations using the $\tau$-test method. A set of relative gravity data in Taiwan was used to test this program using five different choices of command-line arguments. The results show that there are no outliers in these data and that the estimated reading accuracy of the LaCoste and Romberg G meter (serial number 838) is about $0.02 \mathrm{mgal}$ and the gravimeter drift rate is $0.9 \mathrm{mgal} / \mathrm{month}$. The coefficients of the long wavelength and periodic components of calibration function are statistically equal to zero. The result from the datum-free solution is used to detect gravity variation due to the 1999 Chi-Chi earthquake, concluding that only one station experiences a significant change. (C) 2002 Elsevier Science Ltd. All rights reserved.
\end{abstract}

Keywords: Relative gravity; Weighted constraint; Datum free; Gravimeter; Chi-Chi earthquake; Taiwan

\section{Introduction}

Relative gravity measurements are fundamental observations for determining gravity values. It is a common practice to collect redundant observations to ensure a better quality of gravity determination, and hence gravity measurements need to be adjusted using adequate mathematical and stochastic models. Gravity

\footnotetext{
Code available on server at http://www.iamg.org/CGEditor/index.htm or at http://space.cv.nctu.edu.tw/Research/ moi_gravity.htm.

*Corresponding author. Tel.: + 886-3-5724739; fax: + 886-35716257.

E-mail address: hwang@geodesy.cv.nctu.edu.tw

(C. Hwang).

URL: http://space.cv.nctu.edu.tw.
}

measurements may contain gross, random and systematic errors. Systematic errors are primarily due to gravimeter drift and unmodeled calibration factors of instrument reading (e.g., Torge, 1989). In some cases, outliers (gross errors) are hidden in the data, and failure to detect them will result in a false determination of gravity values. Methods for adjusting observations and detecting outliers are abundant in the geodetic literature, e.g., Baarda (1966), Koch (1987), Caspary (1988), and Dudewicz and Mishra (1988). Specific methods for adjusting relative gravity measurements and estimating gravimeter parameters can be found in, e.g., Torge (1989), Lagios (1983), and Jiang et al. (1988).

This paper will first address the problem of rank defect in adjusting relative gravity measurements and then present two adjustment models. One model is called the weighted constraint model, which introduces a priori 
gravity values at given stations as constraints. The other model is called the datum-free model, which employs a datum-free constraint. Methods for detecting outliers will also be presented. Both adjustment models allow the solution of gravimeter parameters such as drift coefficients and coefficients of periodic components. As a case study, relative gravity data collected in a recent survey campaign in Taiwan will be used to test the adjustment program. This survey campaign is primarily for determining whether gravity changes have occurred at 16 gravity stations in Taiwan after the Chi-Chi earthquake, which has a magnitude of 7.3 on the Richter scale (Wang et al., 2000) and occurred on September 21, 1999. All programs described in this paper are coded in FORTRAN 90.

\section{Adjustment theory}

\subsection{Observation equation of relative gravity measurement}

A reading of a relative gravimeter, $l$, at a gravity station, $z$, after multiplying by a conversion factor and correcting for environmental effects (e.g., solid earth tide, ocean tide, air pressure, etc.; see, e.g., Torge, 1989), can be used to form the following observation equation:

$l(t)+v=g+N_{0}+\Delta F(z)+D(t)$,

where $t$ is the time of measurement; $l(t)$ the observed gravity value from multiplication of the counter reading $(z)$ by a calibration factor and corrected for environmental effect; $v$ the residual of $l(t) ; g$ the gravity value at the station; $N_{0}$ the constant bias; $\Delta F(z)$ the calibration function to correct for the error in reading calibration factor; $z$ the gravimeter reading, in counter units (CU); $D(t)$ the drift of gravimeter.

For the stochastic modeling, $\Delta F(z)$ and $D(t)$ in Eq. (1) are termed "systematic errors" due to the gravimeter. Mathematical models for $\Delta F(z)$ and $D(t)$ are well established in the literature. In this paper, $\Delta F(z)$ is modeled as (Torge, 1989)

$\Delta F(z)=\sum_{l=1}^{r} b_{l} z^{l}+\sum_{l=1}^{s}\left(x_{l} \cos \omega_{l} z+y_{l} \sin \omega_{l} z\right)$,

where $b_{l}, x_{l}, y_{l}$ are coefficients, $\omega_{l}$ is the frequency of the reading $z$, and $r, s$ are the numbers of terms to model. The first term on the right side of Eq. (2) is called the long wavelength component, and the second term called the periodic component. For the LaCoste and Romberg G gravimeter, the frequency $\omega_{l}$ can be found in, e.g., Krieg (1981), and Jiang et al. (1988). Furthermore, the drift of a gravimeter $D(t)$ can be modeled by a polynomial as

$D(t)=\sum_{p=1}^{a} d_{p}\left(t-t_{0}\right)^{p}$ where $t_{0}$ is an initial epoch and $a$ is the degree of polynomial ( $a$ depends on the gravimeter characteristics, but rarely exceeds 2 ). The relative gravity observation between stations $i$ and $j, \Delta l_{i j}$, is then

$$
\begin{aligned}
\Delta l_{i, j}+v_{i, j}= & g_{j}-g_{i}+\left(\Delta F\left(z_{j}\right)-\Delta F\left(z_{i}\right)\right) \\
& +\left(D\left(t_{j}\right)-D\left(t_{i}\right)\right),
\end{aligned}
$$

where $v_{i, j}$ is the residual of $\Delta l_{i j}=l_{j}-l_{i}$, and $t_{i}, t_{j}$ are the measurement times. In Eq. (4), $N_{0}$ has been eliminated due to subtraction. Assuming that there are $n$ measurements, the matrix representation of the observation equations is

$\mathbf{L}^{\mathrm{b}}+\mathbf{V}=\mathbf{A X}$

where $\mathbf{L}^{\mathrm{b}}$ is an $n \times 1$ vector of relative gravity measurements, weight matrix is $\mathbf{P} ; \mathbf{V}$ the $n \times 1$ vector of residuals; A the design matrix; $\mathbf{X}$ a $u \times 1$ vector of unknowns, i.e., gravity values $g_{i}$ and gravimeter parameters $b_{l}, x_{l}, y_{l}$ and $d_{p}$ for modeling $\Delta F(z)$ and $D(t)$ and $u$ is the number of unknowns.

\subsection{Least-squares adjustment by weighted constraint}

The standard least-squares solution of Eq. (5) can be obtained by requiring

$\phi=\mathbf{V}^{\mathrm{T}} \mathbf{P V}=$ a minimum,

where $\phi$ is the target function. Using $\partial \phi / \partial \mathbf{X}=0$, one obtains the normal equations

$\mathbf{A}^{\mathrm{T}} \mathbf{P A X}=\mathbf{A}^{\mathrm{T}} \mathbf{P L}^{\mathrm{b}} \quad$ or $\quad \mathbf{N X}=\mathbf{U}$.

Solution of $\mathbf{X}$ using Eq. (7) is not possible without constraints because $\mathbf{A}$ (or $\mathbf{N}$ ) has a rank defect of 1 , which can be explained below. First, let the unknown vector $\mathbf{X}$ be split into the gravity-related part and the gravimeter-related part, so that

$\mathbf{X}=\left[\begin{array}{l}\mathbf{X}_{\mathrm{g}} \\ \mathbf{X}_{\mathrm{I}}\end{array}\right]$,

where $\mathbf{X}_{\mathrm{g}}$ contains gravity values $g_{i}$ and $\mathbf{X}_{\mathbf{I}}$ contains gravimeter parameters $b_{l}, x_{l}, y_{l}$ and $d_{p}$. One can find a non-zero vector $y$ fulfilling

Ay $=0$.

By inspecting Eq. (4) and the structure of $\mathbf{A}$, one finds

$\mathbf{y}^{\mathrm{T}}=c[\underbrace{11 \cdots 1}_{k} \underbrace{00 \cdots 0}_{u-k}]$,

where $c$ is an arbitrary non-zero constant, $k$ is the number of gravity values and $u-k$ is the number of gravimeter parameters. Thus, $y$ is the only element of the null space of the column vectors of $\mathbf{A}$ (Lancaster and Tismenetsky, 1985). So the $u \times u$ matrix $\mathbf{A}^{\mathrm{T}} \mathbf{P A}$ has a rank of $(u-1)$ and is not positive definite. Thus, there is 
an infinite number of solutions of $\mathbf{X}$, which are all statistically biased (e.g., Koch, 1987).

In order to obtain a solution of $\mathbf{X}$, one must hold fixed at least one gravity value during the adjustment, i.e., introduce a gravity datum (or a constraint). The introduction of constraint can be generalized if more than one a priori gravity value is given in the network, i.e., the a priori gravity values can be treated as additional observations so that

$\mathbf{L}_{\mathrm{g}}+\mathbf{V}_{\mathrm{g}}=\mathbf{A}_{\mathrm{g}} \mathbf{X}=\left[\begin{array}{ll}\mathbf{I} & \mathbf{0}\end{array}\right]\left[\begin{array}{l}\mathbf{X}_{\mathrm{g}} \\ \mathbf{X}_{\mathrm{I}}\end{array}\right]$,

where $\mathbf{L}_{\mathrm{g}}$ contains a priori gravity values with weight matrix $\mathbf{P}_{\mathrm{g}}$ and is treated as additional observation vector with residual vector $\mathbf{V}_{g}$ and covariance matrix $\boldsymbol{\Sigma}_{\mathrm{g}}$. In Eq. (11), $\mathbf{P}_{\mathrm{g}}$ is a diagonal matrix. Each of the diagonal elements in $\mathbf{P}_{\mathrm{g}}$ can be: (1) infinite, which means the corresponding a priori gravity value will not be changed after the adjustment, (2) zero, which means the corresponding a priori gravity value is free for determination, and (3) the inverse of variance of the a priori gravity value. That all diagonal elements of $\mathbf{P}_{\mathrm{g}}$ are zero is not acceptable, because this will render the same normal equations as in Eq. (7).

Eqs. (5) and (11) can be used to form augmented observation equations as

$\mathbf{V}=\left[\begin{array}{c}\mathbf{V} \\ \mathbf{V}_{\mathrm{g}}\end{array}\right]=\left[\begin{array}{c}\mathbf{A} \\ \mathbf{A}_{\mathrm{g}}\end{array}\right] \mathbf{X}-\left[\begin{array}{c}\mathbf{L}^{\mathrm{b}} \\ \mathbf{L}_{\mathrm{g}}\end{array}\right]$

with weight matrix $=\operatorname{diag}\left(\mathbf{P}, \mathbf{P}_{\mathrm{g}}\right)$. By minimizing the new target function

$\bar{\phi}=\mathbf{V}^{\mathrm{T}} \mathbf{P V}+\mathbf{V}_{\mathrm{g}}^{\mathrm{T}} \mathbf{P}_{\mathrm{g}} \mathbf{V}_{\mathrm{g}}$,

one obtains the following new normal equations:

$\left(\mathbf{A}^{\mathrm{T}} \mathbf{P} \mathbf{A}+\mathbf{A}_{\mathrm{g}}^{\mathrm{T}} \mathbf{P}_{\mathrm{g}} \mathbf{A}_{\mathrm{g}}\right) \mathbf{X}=\left(\mathbf{A}^{\mathrm{T}} \mathbf{P} \mathbf{L}^{\mathrm{b}}+\mathbf{A}_{\mathrm{g}}^{\mathrm{T}} \mathbf{P}_{\mathrm{g}} \mathbf{L}_{\mathrm{g}}\right)$

Now the normal matrix is positive definite and the estimate of $\mathbf{X}$ can be computed by

$\hat{\mathbf{X}}=\left(\mathbf{A}^{\mathrm{T}} \mathbf{P} \mathbf{A}+\mathbf{A}_{\mathrm{g}}^{\mathrm{T}} \mathbf{P}_{\mathrm{g}} \mathbf{A}_{\mathrm{g}}\right)^{-1}\left(\mathbf{A}^{\mathrm{T}} \mathbf{P} \mathbf{L}^{\mathrm{b}}+\mathbf{A}_{\mathrm{g}}^{\mathrm{T}} \mathbf{P}_{\mathrm{g}} \mathbf{L}_{\mathrm{g}}\right)$.

After solving $\mathbf{X}$, the residual vector $\mathbf{V}$ is then estimated by Eq. (12), and the corrected observations of relative gravity are computed by $\hat{\mathbf{L}}^{\mathrm{a}}=\mathbf{L}^{\mathrm{b}}+\mathbf{V}$. The a posteriori variance of unit weight is computed as

$\hat{\sigma}_{0}^{2}=\frac{\bar{\phi}}{n+r-u}$,

where $r$ is the number of non-zero diagonal elements in $\mathbf{P}_{\mathrm{g}}$. The a posteriori covariance matrix of $\hat{\mathbf{X}}$ is

$\hat{\mathbf{\Sigma}}_{\hat{\mathbf{x}}}=\hat{\sigma}_{0}^{2}\left(\mathbf{A}^{\mathrm{T}} \mathbf{P} \mathbf{A}+\mathbf{A}_{\mathrm{g}}^{\mathrm{T}} \mathbf{P}_{\mathrm{g}} \mathbf{A}_{\mathrm{g}}\right)^{-1}$.

This is called weighted constraint adjustment of relative gravity measurements. The a priori gravity values with non-zero weights are called constraining values. From the definition of $\mathbf{A}_{\mathrm{g}}$ and $\mathbf{P}_{\mathrm{g}}$, it is clear that $\mathbf{A}_{\mathrm{g}}^{\mathrm{T}} \mathbf{P}_{\mathrm{g}} \mathbf{A}_{\mathrm{g}}$ is also a diagonal matrix, whose non-zero diagonal elements are just the weights of the a priori gravity values. If there is only one constraining gravity value, the adjustment is called minimum constraint adjustment.

\subsection{Least-squares solution by datum-free constraint}

Another method to overcome the rank defect problem in solving Eq. (7) is to introduce the so-called datum-free constraint without the need of any a priori gravity values:

$\mathbf{S}^{\mathrm{T}} \mathbf{X}=0$,

where $\mathbf{S}$ is a vector satisfying the condition (Koch, 1987; Caspary, 1988)

$\mathbf{A S}=0$.

Thus the $\mathbf{S}$ vector is equal to $y$ in Eq. (10). For simplicity, we may set $c=1$ in Eq. (10) to get

$\mathbf{S}^{\mathrm{T}}=\left[\begin{array}{llllllll}1 & 1 & \cdots & 1 & 0 & 0 & \cdots & 0\end{array}\right]$.

With Eq. (5) as the observation equations, the leastsquares solution of $\mathbf{X}$, subject to the constraint in Eq. (18) is (Caspary, 1988; Koch, 1987)

$\hat{\mathbf{X}}^{+}=\left(\mathbf{A}^{\mathrm{T}} \mathbf{P} \mathbf{A}+\mathbf{S} \mathbf{S}^{\mathrm{T}}\right)^{-1} \mathbf{A}^{\mathrm{T}} \mathbf{P} \mathbf{L}^{\mathrm{b}}$.

The a posteriori variance of unit weight is

$\hat{\sigma}_{0}^{2}=\frac{\mathbf{V}^{\mathrm{T}} \mathbf{P V}}{n+1-u}$.

With Eq. (21) and the covariance matrix of $\mathbf{L}^{\mathrm{b}}, \hat{s}_{0}^{2} \mathbf{P}^{-1}$, the a posteriori covariance matrix of $\mathbf{X}^{+}$can be obtained by covariance propagation:

$\hat{\mathbf{S}}_{\hat{\mathbf{x}}^{+}}=\hat{\sigma}_{0}^{2}\left(\mathbf{N}+\mathbf{S S}^{\mathrm{T}}\right)^{-1} \mathbf{N}\left(\mathbf{N}+\mathbf{S S}^{\mathrm{T}}\right)^{-1}=\hat{\sigma}_{0}^{2} \mathbf{N}^{+}$,

where $\mathbf{N}^{+}$is the pseudo-inverse of $\mathbf{N}$ (e.g., Caspary, 1988). It can be shown that the trace of $\mathbf{N}^{+}$is the minimum among all generalized inverse of $\mathbf{N}$. Because of this property, the solution by Eq. (21) is called the minimum-trace solution. In addition, the solution in Eq. (21) is also called minimum norm solution because the norm $\left\|\mathbf{X}^{+}\right\|$is minimum among all possible solutions.

Eq. (18) can be written as

$\sum_{i=1}^{k} \hat{g}_{i}=0$

which means that the average of the estimated gravity values is zero. To have a practical use of the solution of $\mathbf{X}^{+}$, we recall that the residual vector $\mathbf{V}$ is estimable (or unique) and hence $\mathbf{L}^{\mathrm{b}}+\mathbf{V}$, which contains the corrected observations, is also estimable without bias. This means that $\mathbf{A} \mathbf{X}^{+}=\mathbf{L}^{\mathrm{b}}+\mathbf{V}$ is estimable. Recalling that

$\hat{\mathbf{X}}^{+}=\left[\begin{array}{c}\hat{\mathbf{X}}_{\mathrm{g}}^{+} \\ \hat{\mathbf{X}}_{\mathrm{I}}^{+}\end{array}\right]$ 
and the definition of A matrix (see Eqs. (4) and (5)), it is clear that the difference between any two estimated gravity values (by Eq. (21)) is estimable. With this understanding, the procedure of datum-free adjustment is then: (a) solve $\mathbf{X}^{+}$by Eq. (21), (b) compute the difference in gravity between any station and a station with a known gravity value using the estimated values in $\mathbf{X}^{+}$, and (c) for each of the stations add the difference from (b) to get the desired gravity value at the station. The gravimeter parameters are also uniquely determined by the adjustment.

The advantages of using datum-free adjustment are

(a) The residuals and corrected observations can be uniquely determined without using any datum constraints such as in Eq. (11).

(b) Relative gravity between two stations can be uniquely determined without datum constraints, thus allowing for monitoring relative gravity change.

\subsection{Global model test and detection of outliers}

The least-squares method is not resistant to outliers in the data, which causes false results in adjustment computations. The choice of mathematical and stochastic models may be incorrect, again leading to false results. Therefore, a global model test must be performed as a routine part of the adjustment. Specifically, if the following condition is met, then the adjustment model is correct and complete:

$\chi^{2}=\frac{R}{\sigma_{0}^{2}}<\chi_{\mathrm{c}}^{2}(1-\alpha ; m)$,

where $\sigma_{0}^{2}$ is the a priori variance of unit weight, $R=\phi$ (for datum-free) or $R=\bar{\phi}$ (for weighted constraint), and $\chi_{\mathrm{c}}^{2}(1-\alpha ; m)$ is the critical value of $\chi^{2}$-distribution when the confidence level of the model test is $(1-\alpha)$ and the degree of freedom of adjustment is $m$. (Note: $\alpha$ is called significance level and is the probability of committing a Type I error). If the condition in Eq. (26) is not met, one should check whether the mathematical and stochastic models are adequate, as well as whether outliers exist in the data. An approximate formula of $\chi_{\mathrm{c}}^{2}(1-\alpha ; m)$ is given by Koch (1987)

$\chi_{\mathrm{c}}^{2}(1-\alpha ; m)=m\left[x_{1-\alpha}\left(\frac{2}{9 m}\right)^{1 / 2}+1-\frac{2}{9 m}\right]^{3}$,

where $x_{1-\alpha}$ is computed by (for $0<\alpha<0.5$ )

$x_{1-\alpha}=t-\frac{2.515517+0.802853 t+0.010328 t^{2}}{1+1.432788 t+0.189269 t^{2}+0.001308 t^{3}}$

and $t=[2 \ln (1 / \alpha)]^{1 / 2}$.
Several methods can be used to detect outliers in observations, e.g., Baarda's data snooping technique (Baarda, 1968) and Pope's $\tau$-test method (Pope, 1976). According to Caspary (1988), these two methods are all efficient in removing outliers. In this paper, Pope's $\tau$-test method is adopted. Pope's $\tau$-test method requires the covariance matrix of residuals, which can be easily obtained by covariance propagation. First, Eq. (12) can be expressed as

$$
\begin{aligned}
& \mathbf{V}=\left[\begin{array}{c}
\mathbf{A} \\
\mathbf{A}_{\mathrm{g}}
\end{array}\right]\left[\begin{array}{ll}
\overline{\mathbf{N}}^{-1}\left(\begin{array}{ll}
\mathbf{A}^{\mathrm{T}} \mathbf{P} & \mathbf{A}_{\mathrm{g}}^{\mathrm{T}} \mathbf{P}_{\mathrm{g}}
\end{array}\right)-\mathbf{I}
\end{array}\right]\left[\begin{array}{l}
\mathbf{L}^{\mathrm{b}} \\
\mathbf{L}_{\mathrm{g}}
\end{array}\right] \\
& =\mathbf{G}\left[\begin{array}{l}
\mathbf{L}^{\mathrm{b}} \\
\mathbf{L}_{\mathrm{g}}
\end{array}\right] \text {, }
\end{aligned}
$$

where $\overline{\mathbf{N}}=\mathbf{A}^{\mathrm{T}} \mathbf{P A}+\mathbf{A}_{\mathrm{g}}^{\mathrm{T}} \mathbf{P}_{\mathrm{g}} \mathbf{A}_{\mathrm{g}}$. Thus the covariance matrix of the combined residual vector $\mathbf{z}=\left[\begin{array}{ll}\mathbf{V} & \mathbf{V}_{\mathrm{g}}\end{array}\right]^{\mathrm{T}}$ in the weighted constraint solution is

$$
\begin{aligned}
& \mathbf{S}_{\mathbf{z}}=\hat{\sigma}_{0}^{2} \mathbf{G}\left[\begin{array}{cc}
\mathbf{P}^{-1} & 0 \\
0 & \mathbf{P}_{\mathrm{g}}^{-1}
\end{array}\right], \\
& \mathbf{G}^{\mathrm{T}}=\hat{\sigma}_{0}^{2}\left[\begin{array}{cc}
\mathbf{P}^{-1}-\mathbf{A} \overline{\mathbf{N}}^{-1} \mathbf{A}^{\mathrm{T}} & -\mathbf{A} \overline{\mathbf{N}}^{-1} \mathbf{A}_{\mathrm{g}}^{\mathrm{T}} \\
-\mathbf{A}_{\mathrm{g}} \overline{\mathbf{N}}^{-1} \mathbf{A}^{\mathrm{T}} & \mathbf{P}_{\mathrm{g}}^{-1}-\mathbf{A}_{\mathrm{g}} \overline{\mathbf{N}}^{-1} \mathbf{A}_{\mathrm{g}}^{\mathrm{T}}
\end{array}\right] .
\end{aligned}
$$

In the datum-free solution, the residual vector can be expressed as

$\mathbf{V}^{+}=\left[\mathbf{A}\left(\mathbf{N}+\mathbf{S S}^{\mathrm{T}}\right)^{-1} \mathbf{A}^{\mathrm{T}} \mathbf{P}-\mathbf{I}\right] \mathbf{L}^{\mathrm{b}}=\mathbf{K} \mathbf{L}^{\mathrm{b}}$.

Using the fact that $\mathbf{A S}=\mathbf{N S}=0$ and matrix equalities (e.g., Lancaster and Tismenetsky, 1985), the covariance matrix of $\mathbf{V}^{+}$is

$$
\begin{aligned}
\mathbf{S}_{\mathbf{V}^{+}} & =\hat{\sigma}_{0}^{2} \mathbf{K} \mathbf{P}^{-1} \mathbf{K}^{\mathrm{T}}=\hat{\sigma}_{0}^{2}\left(\mathbf{P}^{-1}-\mathbf{A} \mathbf{N}^{+} \mathbf{A}^{\mathrm{T}}\right) \\
& =\hat{\sigma}_{0}^{2}\left[\mathbf{P}^{-1}-\mathbf{A}\left(\mathbf{N}+\mathbf{S S}^{\mathrm{T}}\right)^{-1} \mathbf{A}^{\mathrm{T}}\right] .
\end{aligned}
$$

Let $v_{i}$ be the residual of the $i$ th observation. From Eq. (30) or (32), the standard error of $v_{i}, \sigma_{v_{i}}$, can be computed. If

$\frac{\left|v_{i}\right|}{\sigma_{v_{i}}}>\tau_{\mathrm{c}}\left(1-\frac{\alpha}{n} ; 1, m\right)$

then the $i$ th observation is an outlier. In Eq. (33), $n$ is again the number of observations, $\alpha$ and $m$ are the same as those in Eq. (26), and $\tau_{\mathrm{c}}$ is the critical $\tau$ value computed with a confidence level of $(1-\alpha / n)$ and degrees of freedoms of 1 and $m$ (Pope, 1976). A Fortran subroutine to compute $\tau_{\mathrm{c}}$ can be found in Pope (1976). For the weighted constraint solution, the test in Eq. (33) can be used to see whether the constraining gravity values are reasonable and do not damage the result. A constraining value whose residual satisfies Eq. (33) should be removed from the list of constraints. 


\subsection{Test of gravity change}

In this paper, gravity changes due to the Chi-Chi earthquake of Taiwan will be investigated. Here, a testing hypothesis is needed to test whether gravity change is statistically significant at a given station. The testing procedure below is derived from the general hypothesis test in the Gauss-Markoff model, see, e.g., Koch (1987) and Caspary (1988). Let $g_{1}$ and $g_{2}$ be the gravity values measured at two different epochs at the same gravity station. To test whether $g_{1}$ and $g_{2}$ are statistically different, namely, the gravity value has changed from one epoch to another, one can employ the following hypothesis test:

Null hypothesis $\mathrm{H}_{0}: g_{2}-g_{1}=0$,

Alternative hypothesis $\mathrm{H}_{1}: g_{2}-g_{1} \neq 0$.

If the test statistic $T$ satisfies

$T=\frac{\left|g_{2}-g_{1}\right|}{\sqrt{\sigma_{1}^{2}+\sigma_{2}^{2}}}>t_{\mathrm{c}}(1-\alpha ; m)$,

then $\mathrm{H}_{0}$ is rejected and the change $\left(g_{2}-g_{1}\right)$ is statistically significant. A typical value of $\alpha$ is 0.05 . In Eq. (34), $\sigma_{1}$ and $\sigma_{2}$ are the standard errors of $g_{1}$ and $g_{2}$, respectively, $m$ is the sum of the degrees of freedom in determining $g_{1}$ and $g_{2}$, and $t_{\mathrm{c}}(1-\alpha ; m)$ is the critical $t$-value when the two-tailed significance level is $\alpha$ and the degree of freedom is $m$. In many cases, the previous gravity value $g_{1}$ was determined long time ago and the method to compute $g_{1}$ is unclear, thus the degree of freedom and the standard error associated with $g_{1}$ cannot be well estimated and the testing result can be unreliable.

\section{FORTRAN 90 programs for adjustment and test of gravity change}

A program, called gravnet.f90, in FORTRAN 90 is developed for adjustment of relative gravity measurements and estimation of gravity values and gravimeter parameters using weighted and datum-free constraints (Section 2.2 and 2.3). This program accepts commandline arguments and is suitable for the UNIX environment and the Microsoft DOS mode. A description of this program, which also appears as comments in the program unit, is presented below:

\section{Name}

gravnet-adjust relative gravity measurements and estimate gravities and gravimeter parameters, in FORTRAN 90

\section{Synopsis}

gravnet -Dname1.obs -Nname2 [-Aid -Ccdegree Ffixed_stn_file -Lsignificance_level -Mmodel -Pperiod_ file -Tdegree-Smjd0]

\section{Description}

D file of observations

$\mathrm{N}$ "name2" will be used as the prefix of the following output files:

name2.gra: estimated gravity values and standard deviations

name2.met: gravity meter drift and calibration

parameters

name2.res: residuals of observations and test result

name2.err: outliers (gross errors)

name2.sta: summary of residuals and standard

deviations of estimated gravities

name2.his: histogram of residuals

\section{Options}

A use id_th station as the starting gravity for-M1-F [default: 1]

$\mathrm{C}$ model long wavelength calibration function up to degree cdegree, see "P"; will model only long wavelength part if $\mathrm{P}$ is not specified [default: do not model calibration function]

F file of fixed stations, contains station name, gravity value and standard deviation when choosing weighted constraint model, this option must be activated

$\mathrm{L}$ significance level of global model test and $\tau$-test of observations (typically 5\%)

$\mathrm{M}$ type of adjustment model. 1 = datum-free, $2=$ weighted constraint [default: 1]

$\mathrm{P}$ model the periodic components of calibration function. This file contains the periods (in $\mathrm{CU}$ ) of the functions to be modeled.

[default: no such components]

$\mathrm{S}$ initial epoch in modified Julian Day (MJD) for modeling drift [default: MJD at the first measurement time]

T polynomial degree of gravity meter drift [default: 1]

Important notes on program gravnet.f90 are

(1) The contents of the file of relative gravity measurements are

Record 1: maximum possible number of gravity stations

Record 2: additional information

Record 3 to last record: All contain starting station (start), ending station (end), difference (mGal), MJD (start), MJD (end), reading (start), reading (end), standard error of difference (mGal).

Here readings are in CU, MJD at measurement time (see, e.g., Seeber, 1993), and difference $\left(\Delta l_{i j}\right.$ in Eq. (3)) is relative gravity measurement as obtained by differencing the reduced gravity values at the ending and starting stations. Subroutine DATE2MJD in gravnet.f90 will compute MJD based on the calendar date.

(2) The datum-free and minimum constraint solutions will lead to the same result if the starting gravity value for the former is the same as the constraining gravity value for the latter. 
(3) To model the periodic components of calibration function (the P option), the user must supply a file which contains line-by-line the periods of reading in $\mathrm{CU}$.

(4) The critical $\tau$ value in Eq. (33) is automatically computed by subroutine TAURE.

(5) When datum-free is chosen and a file of constraining gravity values is specified (M1, F), the estimated gravity values are derived from the first gravity value (which is called starting gravity value) in the file.

A program, called compare. 990 , is also developed to test whether the gravity change at a given station is statistically significant. A description of this program is as follows:

\section{Name}

compare-compare gravity values at two epochs and test whether change occurs

Synopsis

compare -Anew_gravity -Bold_gravity -Cdifference Lsig_level -Idof

Description

A file of new gravity values
B file of old gravity values

$\mathrm{C}$ file of differences and test result

L significance level (two-tailed)

I degree of freedom

The critical $t$-value $t_{\mathrm{c}}(1-\alpha ; m)$ in Eq. (34) is computed by the function "TIN" from the IMSL numerical libraries.

\section{A case study in Taiwan}

\subsection{A recent survey campaign of relative gravity in Taiwan}

In order to determine the gravity changes at 16 gravity stations in Taiwan thought to be due to the Chi-Chi earthquake, the Ministry of Interior (MOI) of Taiwan sponsored a survey campaign in late 2000 to determine new gravity values at these 16 gravity stations. The locations of the 16 stations are shown in Fig. 1. Before September 21, 1999, the gravity values at six of the 16 gravity stations (Hsinchu, Taichung, Dashi, Hualien,

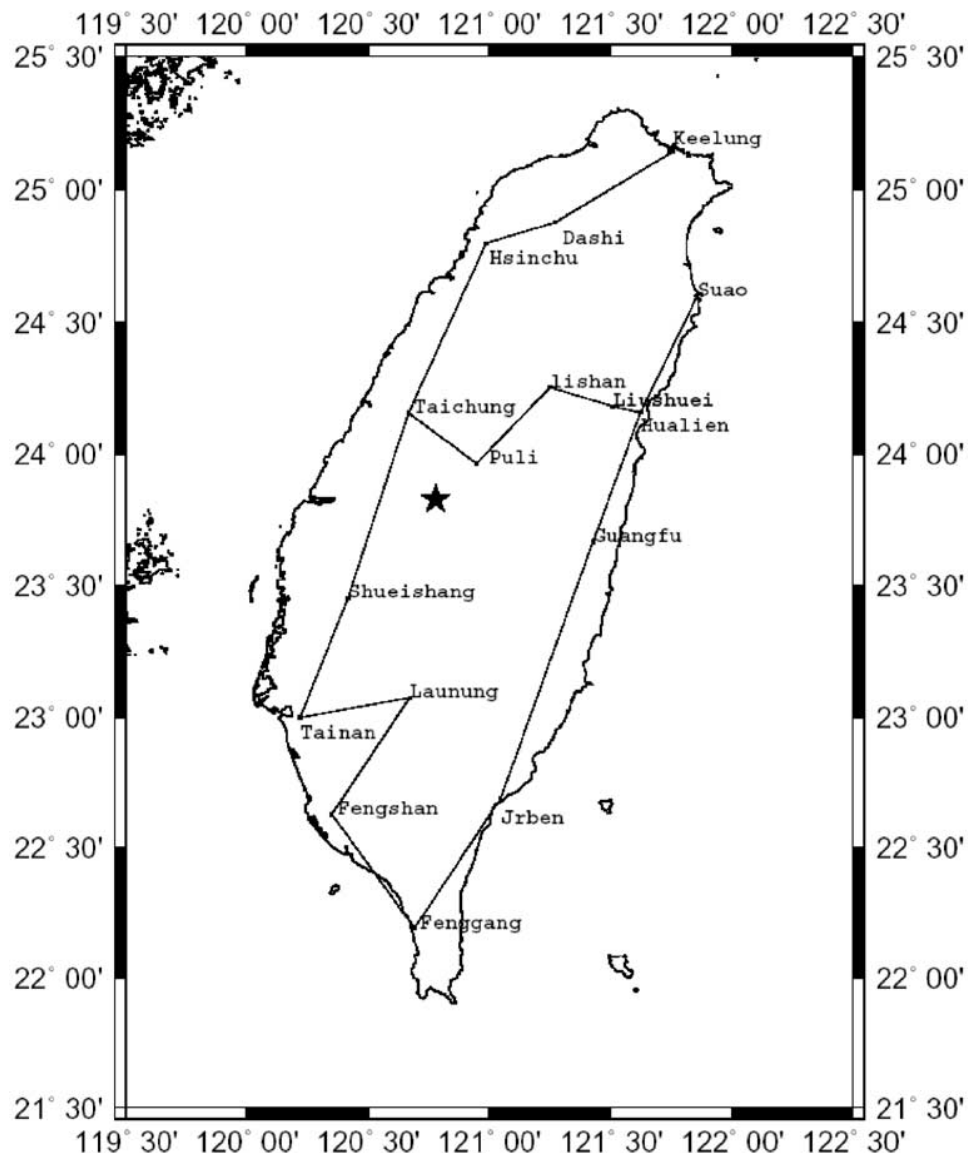

Fig. 1. Locations of 16 gravity stations where relative gravity measurements are collected. Star indicates the epicenter of Chi-Chi earthquake. Lines connecting stations are primary routes of gravity survey. 
Liushuei and Fengshan) were measured using a FG5 absolute gravimeter (see http://www.microgsolutions.com/fg5.htm), with standard errors ranging from 2 to $3 \mu$ gal $\left(10^{-8} \mathrm{~m} / \mathrm{s}^{2}\right)$. These stations are called absolute gravity stations. The gravity values at the other 10 stations were determined by a LaCoste and Romberg $G$ gravimeter and their standard errors are about 30-50 $\mu$ gal (Chang, 1999).

In the MOI-sponsored campaign, a LaCoste and Romberg $G$ gravimeter (serial number 838 ) was used to collect a total of 56 relative gravity values in about two weeks. The raw gravity data were corrected for the effects of solid earth tide, ocean tide, pressure change and polar motion using the formulae found in Torge (1989), Van et al. (1986), and Moritz, Mueller (1987). Considering the $40 \mu \mathrm{gal}$ accuracy of LaCoste and Romberg G meter (LCR, 2001), only the solid earth tide correction, which has a maximum absolute value of about $0.3 \mathrm{mgal}$, is significant. The other three corrections amount to only few $\mu$ gal, which is far smaller than the $G$ gravimeter measuring error. In particular, the effects of pressure change and polar motion have periods longer than a month, hence such effects will be largely eliminated when forming relative gravity measurement using Eq. (4). The measuring lines in this campaign form a closed loop around Taiwan, with a line running through the Central Range (see Fig. 2). Such a strong geometry of measurements is expected to result in a uniform accuracy of gravity values.

\subsection{Results of adjustments}

The 56 relative gravity measurements described above have been used for data analysis. A total of five cases were tested. A description of the five cases and the command-line arguments associated with these cases are as follows:

Case 1: datum-free, use the first gravity value in file six.fix to the starting value, model drift to degree one gravnet -Dtaiwan.obs -Ncase1 -M1 -Fsix.fix -T1.

Case 2: same as Case 1, but also model long wavelength and periodic components of calibration function with periods from file period.dat

gravnet -Dtaiwan.obs -Ncase2 - -M1 - -Fsix.fix $-\mathrm{C} 1$ -Pperiod.dat -T1.

Case 3: weighted constraint, use the six gravity values in file six.fix as constraints, model long wavelength component of calibration function and drift to degree one gravnet -Dtaiwan.obs -Ncase3 -M2 -Fsix.fix -C1 -T1.

Case 4: weighted constraint, use the six gravity values in file six.fix as constraints, model periodic components of calibration function and drift to degree one gravnet -Dtaiwan.obs -Ncase4 -M2 -Fsix.fix -Pperiod. dat $-\mathrm{T} 1$

Case 5: weighted constraint, use the six gravity values in file six.fix as constraints, model long wavelength and periodic components of calibration function and drift to degree one

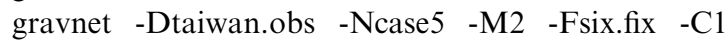
-Pperiod.dat -T1.

The file taiwan.obs contains 56 observations, the file period.dat contains two periods 36.67 and 73.33 (in CU), which are taken from Jiang et al. (1988), and the file six.fix contains the six a priori gravity values at the six absolute gravity stations. Because of the relatively short time of data collection, the gravimeter drift and long wavelength components are modeled only up to degree one. In Cases 1 and 2, the Dashi gravity value is used as the starting gravity value to compute the gravity values at the other stations using the estimated gravity differences. The Dashi station is far from the epicenter of the Chi-Chi earthquake (see Fig. 1) and its gravity value is assumed to remain unchanged after this earthquake.

Table 1 summarizes the results from the five cases. Since in Cases 1 and 2 the Dashi gravity value is assumed to be errorless, the minimum standard errors (for the estimated Dashi gravity value) in these cases are of course zero. Table 2 summarizes the estimated parameters of the LaCoste and Romberg G gravimeter that was used in the data collection. All cases yield a consistent accuracy of gravimeter reading of about $0.02 \mathrm{mgal}$, which is better than the claimed accuracy of 0.04 mgal by LCR (2001). In all cases, the gravimeter drift is estimated to be about $0.9 \mathrm{mgal} / \mathrm{month}$, which is consistent with the published drift by LCR (2001). Results of global model tests by Eq. (26) show that all cases use adequate adjustment models. Fig. 2 shows the histograms of residuals from the five cases. The residuals almost follow a normal distribution, which suggest that in all cases there is no significant systematic effect in the adjustment models.

For all cases, the $\tau$-tests show no outliers in the relative gravity data, and the six constraining gravity values are all adequate. However, the standard errors of the estimated gravity values from Case 2 are relatively large (see Table 2), which is caused by the high correlation between coefficient $b_{l}$ (see Eq. (2)) and estimated gravity values. Because of this high correlation, it is difficult to separate coefficient $b_{l}$ and estimated gravity values, and it is not recommended to use the datum-free adjustment when coefficient $b_{k}$ is sought.

To see whether an estimated parameter $p$ of the gravimeter is statistically equal to zero, the following hypothesis was tested:

Null hypothesis $\mathrm{H}_{0}: p=0$

Alternative hypothesis $\mathrm{H}_{1}: p \neq 0$

Let $\hat{p}$ and $\hat{\sigma}$ be the estimated value of a gravimeter parameter and its standard error, $\alpha$ be the two-tailed significance level. If

$\frac{|\hat{p}|}{\hat{\sigma}}<t_{\mathrm{c}}(1-\alpha ; m)$ 
Case 1

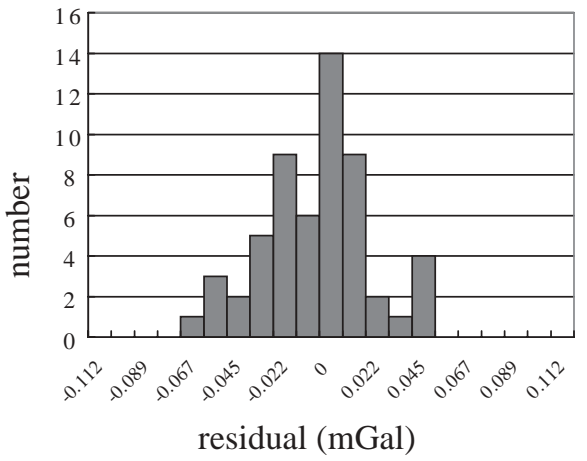

Case 3

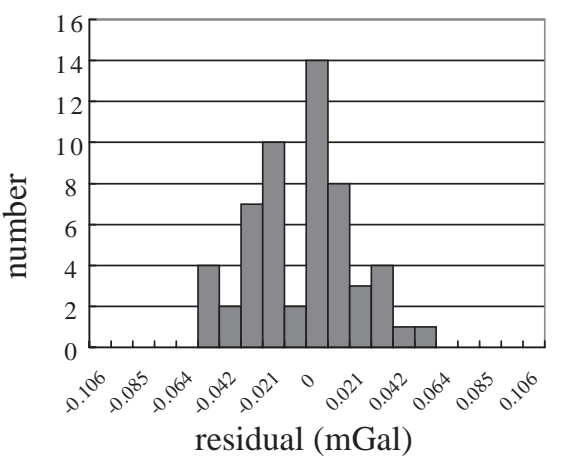

Case 2

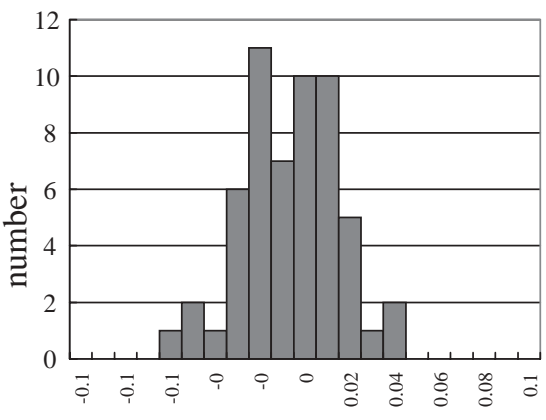

residual (mGal)

Case 4

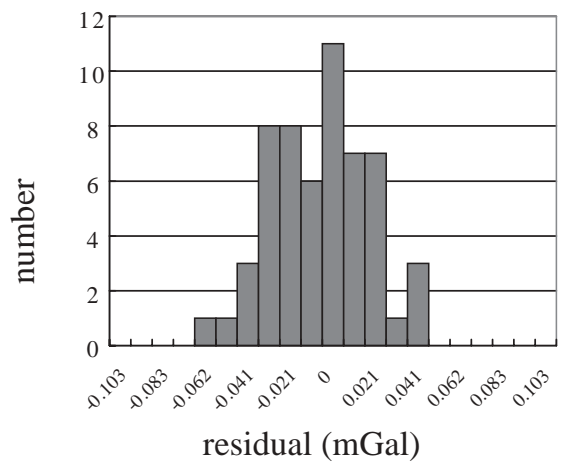

Case 5

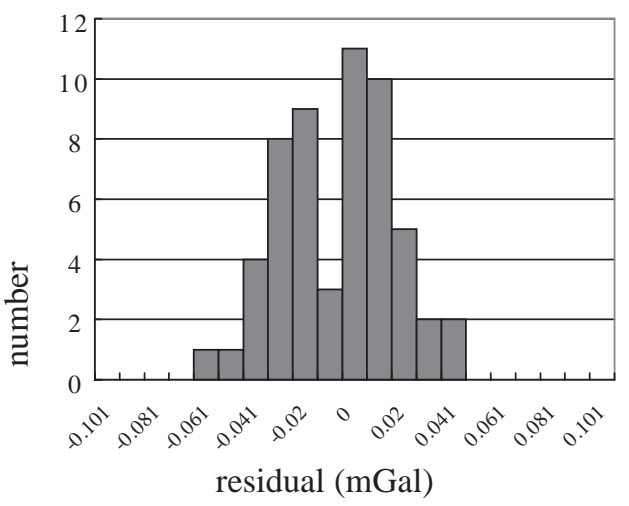

Fig. 2. Histograms of residuals from five adjustment cases.

then $\mathrm{H}_{0}$ is accepted and this gravimeter parameter is statistically equal to zero. It was found that for all cases, the estimated gravimeter parameters are statistically equal to zero. This means that we do not need to model gravimeter parameters for the current gravity data set (except modeling drift, which is suggested by the manufacturer of the $\mathrm{G}$ gravimeter). That is, for the current data set, Case 1 will fulfill the goal of adjusting relative gravity measurements and estimating gravity values. However, for a large network and a long time span of data collection, it is suggested to estimate gravimeter parameters using the weighted constraint model with constraining stations evenly distributed in the network.

\subsection{Result of testing gravity change}

It is clear that if one wishes to detect gravity change in a network, one cannot constrain any a priori gravity value in the network during the adjustment, except one 
Table 1

Summary of results of five adjustment cases (units in mgal)

\begin{tabular}{|c|c|c|c|c|c|c|c|}
\hline Case & Global test & Outlier & Maximum residual & Minimum residual & $\begin{array}{l}\text { Maximum std. } \\
\text { dev. of gravity }\end{array}$ & $\begin{array}{l}\text { Minimum std. } \\
\text { dev. of gravity }\end{array}$ & $\begin{array}{l}\text { RMS std. dev. } \\
\text { of gravity }\end{array}$ \\
\hline 1 & Pass & No & 0.0550 & -0.0567 & 0.0452 & 0.0000 & 0.0287 \\
\hline 2 & Pass & No & 0.0476 & -0.0510 & 1.1519 & 0.0030 & 0.5911 \\
\hline 3 & Pass & No & 0.0560 & -0.0500 & 0.0539 & 0.0025 & 0.0167 \\
\hline 4 & Pass & No & 0.0466 & -0.0568 & 0.0536 & 0.0025 & 0.0223 \\
\hline 5 & Pass & No & 0.0487 & -0.0526 & 0.0899 & 0.0024 & 0.0309 \\
\hline
\end{tabular}

Table 2

Estimated parameters of LaCoste and Romberg G gravimeter (serial number 838) from five adjustment cases

\begin{tabular}{llllrr}
\hline Parameter & Case 1 & Case 2 & Case 3 & Case 4 & Case 5 \\
\hline Single reading accuracy (mgal) & \pm 0.023 & \pm 0.020 & \pm 0.021 & \pm 0.021 & \pm 0.021 \\
Linear drift (mgal/day) & $-0.030 \pm 0.055$ & $-0.032 \pm 0.048$ & $-0.031 \pm 0.051$ & $-0.031 \pm 0.050$ & $-0.031 \pm 0.050$ \\
$b_{1}$ (mgal/CU) & $\mathrm{NC}$ & $-0.001 \pm 0.003$ & $-0.000 \pm 0.000$ & $\mathrm{NC}$ & $-0.000 \pm 0.000$ \\
$x_{1}, y_{1}$ (mgal) periods: $36.67,73.33(\mathrm{CU})$ & $\mathrm{NC}$ & $-0.151 \pm 0.215$ & $\mathrm{NC}$ & $0.039 \pm 0.017$ & $0.013 \pm 0.024$ \\
& & $0.036 \pm 0.083$ & & $-0.040 \pm 0.024$ & $-0.002 \pm 0.035$ \\
& & $-0.023 \pm 0.185$ & & $0.102 \pm 0.037$ & $0.039 \pm 0.055$ \\
& & $-0.571 \pm 0.360$ & & $0.001 \pm 0.011$ & $-0.010 \pm 0.013$ \\
\hline
\end{tabular}

NC: not computed.

value that is for the starting value or for the minimum constraint. Therefore, the result from Case 1 was used to test whether the differences between the new and the old gravity values at the 16 gravity stations (see Fig. 1) are statistically significant. In the test, a two-tailed significance level of $\alpha=0.05$ was used. Table 3 shows the result of the test, which is summarized below.

(1) The gravity values at the six absolute gravity stations remain unchanged (note: one absolute gravity station, Dashi, has been assumed to be unchanged in Case 1 and serves as the starting gravity value). Therefore, use of any of the six stations as the starting gravity value in Case 1 will lead to the same conclusion of no gravity change.

(2) The gravity values at the Suao, Lishan, Keelung and Shueishang stations are found to change. But after a careful inspection, the changes at Suao, Lishan, Keelung should be due to man-made motions of gravity stations. This issue is currently under investigation.

(3) The gravity change of $0.142 \mathrm{mgal}$ at Shueishang is real and may be due to the aftershock (6.5 on the Richter scale) of the Chi-Chi earthquake.

(4) Surprisingly, the gravity values at the Taichung and Puli stations, which are quite close to the epicenter of the Chi-Chi earthquake, remain unchanged. This somewhat contradicts what has been expected of gravity variation near the epicenter.
Table 3

Differences between new and old gravity values and test result

\begin{tabular}{lrrl}
\hline Station & \multicolumn{2}{c}{ Difference $(\mathrm{mGal})$} & Test statistic \\
& & \\
& & \\
Shueishang & -0.1420 & -2.860 & Yes \\
Tainan & -0.0315 & -0.722 & No \\
Launung & 0.0053 & 0.092 & No \\
Taichung & -0.0289 & -0.868 & No \\
Puli & -0.0370 & -0.904 & No \\
Lishan & -0.3661 & -6.013 & Yes \\
Jrben & -0.0282 & -0.622 & No \\
Guangfu & 0.0283 & 0.647 & No \\
Hualien & -0.0332 & -1.128 & No \\
Liushuei & -0.0062 & -0.231 & No \\
Suao & -1.1115 & -24.129 & Yes \\
Fengshan & -0.0096 & -0.211 & No \\
Keelung & -1.0342 & -27.446 & Yes \\
Dashi & 0.0000 & 0.000 & No \\
Hsinchu & -0.0586 & -1.901 & No \\
Fenggang & 0.0123 & 0.225 & No \\
\hline
\end{tabular}

${ }^{\mathrm{a}}$ See Eq. (34).

Regarding (4), Fig. 3 shows the horizontal velocities measured after the Chi-Chi earthquake at selected stations near central Taiwan. Such horizontal motions may cause gravity changes. The relationship between gravity changes and land deformations in central Taiwan is currently under study. It must be emphasized that the current result of gravity change detection is 


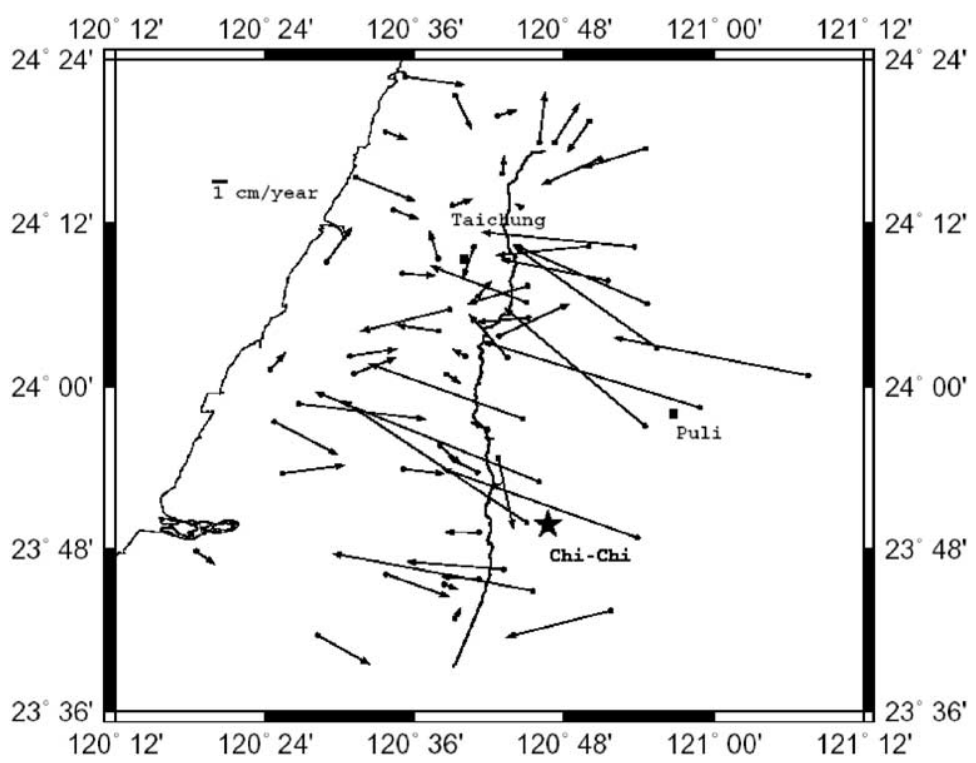

Fig. 3. Horizontal velocities at selected stations in central Taiwan. Star indicates epicenter of Chi-Chi earthquake. Thick line represents Chelungpu Fault that caused Chi-Chi earthquake. Land Surveying Bureau of Taiwan supplied velocities.

based on the data collected by a LaCoste and Romberg $\mathrm{G}$ gravimeter, which has an accuracy of only $0.02 \mathrm{mgal}$. Gravity change below this value is unlikely to be detected.

\section{Conclusions}

A self-contained program for adjusting relative gravity measurements is developed in this paper. The adjustment model can be either weighted constraint or datum-free, depending on data and specific requirements. The weighted constraint model is chosen when there exist reliable a priori gravity values for use as additional data at stations in a network, or when it is required that a gravity network be "attached" to an existing gravity network of a higher order. The datumfree model requires no gravity datum and uniquely determines relative gravity values among all stations. This program was used to adjust recently collected relative gravity measurements in Taiwan. The adjustment results indicate no significant drift and calibration function error of the LaCoste and Romberg G gravimeter used to collect the data. Comparison of the new and old gravity values at 16 stations shows no particular pattern of gravity change due to the Chi-Chi earthquake. The computer programs and test data set described in this paper are available at the WWW site http://space.cv.nctu.edu.tw/Research/ moi_gravity.htm.

\section{Acknowledgements}

This study is supported by the Ministry of Interior, Republic of China (ROC), under the project "Gravity survey on the first-order benchmarks." We are grateful to F.S. Ning of Base Survey Battalion, ROC for data collection.

\section{References}

Baarda, W., 1966. Statistical concepts in geodesy. Netherlands Geodetic Commission, Publications on Geodesy, Delft, Vol. 2(4), 74pp.

Baarda, W., 1968. A testing procedure for use in geodetic network. Netherlands Geodetic Commission, Publications on Geodesy, Delft, Vol. 2(5), 97pp.

Caspary, W.F., 1988. Concept of network and deformation analysis. Monograph 11, School of Surveying, University of New South Wales, Kensington, 183pp.

Chang, R.-G., 1999. Gravity determination at absolute gravity stations and establishment of first-order gravity stations in Taiwan. Report. No. 88-050, Ministry of Interior of Taiwan, Taipei, 118pp (in Chinese).

Dudewicz, E., Mishra, S.N., 1988. Modern Mathematical Statistics. Wiley, New York, 838pp.

Jiang, Z., Zuo, C., Qiu, Q., Xu, S., 1988. China gravity basic net 1985. Scientia Sinica (Series B) 31 (9), 1143-1152.

Koch, K.-R., 1987. Parameter Estimation and Hypothesis Testing in Linear Models. Springer, Berlin, 378pp.

Krieg, L.A., 1981. Mathematical modeling of the behavior of the LaCoste and Romberg " $G$ " gravity meter for use in 
gravity network adjustments and data analyses. Report. No. 321, Ohio State University, Columbus, USA. 186pp.

Lagios, E., 1983. A Fortran IV program for a least-squares gravity base-station network adjustment. Computers \& Geosciences 10, 263-276.

Lancaster, P., Tismenetsky, M., 1985. The Theory of Matrices, 2nd Edition. Academic Press, New York, 570pp.

LCR, 2001. Gravity meter manuals for LaCoste and Romberg models G and D. http://www.LaCosteRomberg.com.

Moritz, H., Mueller, I.I., 1987. Earth Rotation. Ungar, New York, 617pp.
Pope, A.J., 1976. The statistics of residuals and the detection of outliers. Technical Report no. S65 NGS1, Rockville, Maryland, 133pp.

Seeber, G., 1993. Satellite Geodesy. deGruyter, Berlin, $531 \mathrm{pp}$.

Torge, W., 1989. Gravimetry. deGruyter, Berlin, 465pp.

Vanícek, P., Krakiwsky, E., 1986, Geodesy, the Concepts. Elsevier, Amsterdam, 697pp.

Wang, C.S., Hsu, S.K., Kao, H., Wang, C.Y. (Eds.), 2000. Special issue on the 1999 Chi-Chi earthquake in Taiwan. Terrestrial Atmospheric and Oceanic Sciences, 11(3). 